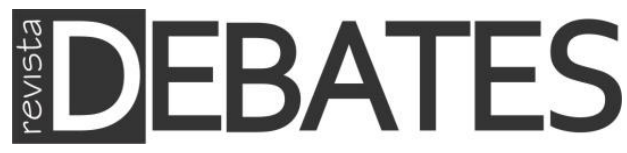

\title{
El (no) estudio de las militancias partidistas
}

\author{
The absence of studies of party militancies
}

\section{Alberto Espejel Espinoza}

\section{Resumen}

El trabajo muestra las diversas miradas sobre las militancias que han prevalecido en la literatura referida a los partidos, igualmente llama la atención sobre la necesidad de efectuar trabajos empíricos. El alcance del trabajo es acomodar la literatura existente, clarificando sus aportes y limitaciones. Por ende, se acomodó la literatura referida a partidos (tanto la enfocada sobre democracia interna que idealiza al militante, presuponiendo su homogeneidad; así como la referida a la militancia que en el contexto latinoamericano es realmente escasa), con base en dónde colocan su atención (estatutos o realidad partidaria) y cuál es su interés (comparar o indagar en profundidad).

\section{Palabras clave}

Democracia Interna; Militancia; Partidos Políticos.

\section{Abstract}

This work shows the different points of view on the militancies that have prevailed in the literature on party politics. It also calls attention to the need to perform empirical work. The scope of this work is to accommodate the existing literature, clarifying its contributions and limitations. Thus, the literature on party politics was adapted (both its segment focused on internal democracy, which idealizes the militant by presupposing its homogeneity, as well as the one referring to a militancy which is actually scarce in the Latin American context), based on where they place their attention (statutes or the reality of parties) and their goals (comparing or inquiring in depth).

\section{Keywords}

Intra-Democracy; Militancy; Political Parties. 


\section{Introducción ${ }^{1}$}

Los partidos políticos han sido estudiados en cuanto a su dinámica interna desde inicios del siglo XX, ejemplo de ello fueron los trabajos de Ostrogorski (1982) y Michels (1983) que dieron inicio a la perspectiva organizativa que ha sido prolija en mostrar la subordinación del militante al dirigente y de éste a la organización.

Sin embargo, los cambios en la sociedad iniciados desde la década de 1960 y hasta la de $1990^{2}$, supusieron la entrada a un tipo de democracia y partidos distintos a los de hace más de medio siglo que nos relató Duverger $(1984)^{3}$. De ahí que se transitó de una democracia de masas con partidos ideológicos y militancias estables a una de corte electoral con interés en el mayor número de ciudadanos, con la consecuente relajación de las ideologías, así como militancias reducidas (KIRCHHEIMER, 1980; KATZ y MAIR, 2007).

Una de las miradas recientes para dar cuenta del funcionamiento de los partidos, hermanada con la perspectiva organizativa, es la relativa a la democracia interna, dicha literatura emergió en el caso mexicano a finales de la década de 1990. Y es que, en esa misma década la oposición cobró importancia y, con ello, el análisis de la vida partidista. Por ende, a lo largo del siglo XXI diversos trabajos han dado evidencia del enorme peso de las dirigencias partidistas en la selección de los dirigentes nacionales y candidatos presidenciales.

No obstante, la gran mayoría estos trabajos parten de un supuesto que ha implicado centrar la mirada en un solo aspecto de los partidos, lo cual ha dejado de lado otras aristas importantes. Dicho supuesto se puede resumir como sigue: para entender cómo funcionan los partidos hay que describir/explicar cómo actúan los dirigentes, cómo se agrupan en torno a tendencias o facciones y cómo se incrustan en las dirigencias. La consecuencia de ello es que se ha idealizado al militante, de ahí que subyace la idea de una militancia desprotegida, por lo cual es necesario implementar

\footnotetext{
1 Investigación realizada gracias al Programa de Apoyo a la Investigación para el Desarrollo y la Innovación (PAIDI) de la FES Acatlán de la UNAM. Proyecto "Las organizaciones partidistas en México: Democracia interna y militancia”.

${ }^{2}$ El auge de los medios de comunicación masiva, la aparición de nuevos movimientos sociales, la pérdida de confianza en las instituciones, la volatilidad electoral, la desideologización política, entre otros (MARTÍNEZ, 2016).

${ }^{3}$ Sobre todo en el caso del partido de masas, el cual contaba con una militancia elevada que acudía a las reuniones de la organización para deliberar, decidir la línea política y las plataformas, así como seleccionar sus candidatos, aunado a que exigía cuentas derivado de que el financiamiento del partido provenía de su bolsillo (DUVERGER, 1984).
} 
mecanismos para la reforma interna y, después de ello, ésta se volcará a tomar decisiones, integrar dirigencias y controlar dirigentes.

Sin embargo, lo anterior pasa por alto que el cambio en la sociedad, en la democracia y en los partidos no es ajeno a las militancias. Por ende, la literatura emergente y especializada en la militancia, emanada de democracias consolidadas, da cuenta de militancias desarraigadas y desinteresadas de la vida interna, salvo en lo que toca a los intereses selectivos (cargos, prebendas, dinero, etc.). En México, así como América Latina, pocos son los trabajos existentes sobre militancias. Por consiguiente, el presente trabajo muestra el estado de la cuestión de la militancia partidista, con el objetivo de alentar trabajos en el contexto mexicano sobre las militancias para complejizar la comprensión que tenemos sobre nuestras organizaciones partidistas. Para lograrlo se llevó a cabo una revisión de literatura relativa a los partidos, tanto los trabajos clásicos, como aquellos englobados bajo el concepto de democracia interna y aquellos que se centran propiamente hablando en el militante ${ }^{4}$.

El texto se divide en cuatro apartados. En primer lugar, se muestra una breve discusión sobre la perspectiva organizativa del estudio de los partidos. En segundo lugar, se presentan los principales aportes derivados de la literatura centrada en la democracia interna, en clave del descuido que subyace en ella al tratar empíricamente al militante. El tercer lugar, se muestran los principales aportes respecto al estudio de la militancia en democracias consolidadas, así como el caso latinoamericano. Finalmente, se concluye con una discusión respecto a la importancia del estudio de las militancias, así como algunas claves de análisis para el caso mexicano.

\footnotetext{
${ }^{4}$ En el primer caso, principalmente se revisaron obras clásicas sobre el estudio de los partidos. Mientras que, en el caso del segundo y tercer tipo de literatura, se hizo una revisión a través de bases de datos, tal como EBSCO, JSTOR, SAGE, Oxford Journals, así como Taylor \& Francis, luego de lo cual se procedió a su acomodo con base en dónde colocan su atención (estatutos o realidad partidaria) y cuál es su interés (comparar o indagar en profundidad). Cabe aclarar que este trabajo es un primer acercamiento al estudios de las militancias, en adelante se desarrollarán estudios con mayor profundidad, analizando el perfil de la militancia, así como su percepción sobre los procesos de toma de decisión al interior de su organización, enfocados en el caso mexicano y cada uno de los nueve partidos con registro oficial de 2015 a 2018: Partido Acción Nacional, Partido Revolucionario Institucional, Partido de la Revolución Democrática, Partido del Trabajo, Partido Verde Ecologista de México, Movimiento Ciudadano, Partido Nueva Alianza, Partido Encuentro Social y Movimiento Regeneración Nacional.
} 


\section{El estudio de los partidos desde la visión clásica organizativa}

Como apuntan Montero y Gunther (2007), existen distintas formas de abordar a los partidos, tal es el caso de las perspectivas ideológica, funcionalista, racional y organizativa ${ }^{5}$. Sin duda, cada una de ellas ha aportado bastante a la comprensión de los partidos, empero la última ha tenido mayor repercusión al pensar a los militantes.

Dicha perspectiva se ha interesado en buena medida, a diferencia de las anteriores, por el conjunto de actores, procesos y reglas que subyacen a un partido. $Y$ uno de los problemas que le ha interesado ha sido el problema de la concentración de poder.

Como apunta Espejel (2015), lo anterior surgió desde los multicitados trabajos de Ostrogorski (1982) y Michels (1983), de hace más de un siglo. En ambos, existió un diagnóstico pesimista de la relación existente entre el dirigente y el dirigido. Ahora bien, dentro de esta perspectiva existió un fuerte ímpetu de pensar a los partidos en su complejidad interna y en su interrelación con el exterior. Panebianco (1990), sin duda, es un autor obligado al respecto, aunque no el pionero. Antes del italiano, emergieron diversos tipos de organizaciones partidistas que al tiempo que teorizaban la relación entre el dirigente y el dirigido, permitían pensar la interrelación del partido con otros actores en diversas arenas.

De ahí que, dichos tipos de partido daban cuenta de la relación del partido con la militancia, el legislativo, las funciones de gobierno, el electorado, entre otros. Pese a ello, en la literatura organizativa subyace la idea de la existencia y prevalencia de un centro de poder, del cual proceden las principales decisiones de la organización.

Retomando el trabajo de Michels (1983), dicho centro de poder se materializa en la oligarquía. Es decir, un grupo compacto de dirigentes que se alejan de la base, debido a motivos técnico-administrativas que derivan en la delegación de tareas, a lo

\footnotetext{
${ }^{5}$ En el caso de la mirada ideológica, cuyo máximo representante fue Von Beyme (1986), el interés por la organización estriba en la prevalencia de valores y creencias que militantes y dirigentes comparten y defienden. No obstante, como consecuencia del llamado fin de las ideologías y el ocaso del party government dicha mirada entró en desuso. Mientras que la visión funcionalista, surgida en los sesenta, centró su interés en las funciones que cumplían los partidos (SARTORI, 1980), tales como: agregar y articular intereses, reclutar, así como la socialización y comunicación de políticas. Empero, el auge de los mass media arrebató a los partidos las dos últimas funciones. Mientras que, la perspectiva racional ha considerado que los partidos son actores racionales que no están interesados en defender el bienestar público, sino que más bien pretenden alcanzar los cargos (DOWNS, 1973). Una de las principales críticas a tal enfoque es no haber considerado la enorme variedad de tipos de partidos existentes, así como su complejidad interna.
} 
cual le sigue la profesionalización del dirigente, la incompetencia de la militancia que necesita y venera líderes, así como la transformación de los dirigentes en actores autointeresados ${ }^{6}$.

Entretanto, en la obra de Duverger también puede rastrearse la idea de un centro de poder. Así, en el caso del partido de cuadros se trata del grupo de parlamentarios, un auténtico grupo de notables, quienes detentan el poder, ya que las actividades fundamentales de la organización son acudir a elecciones y participar en el parlamento. Mientras que, en el partido de masas, el centro de poder recae en la dirigencia que es apoyada por la militancia activa y numerosa que financia al partido, y logra imponerse al partido en el gobierno.

Más adelante, Kirchheimer (1980) mostraría que los partidos habrían mutado derivado de los cambios sociales, económicos, culturales y políticos de mediados del siglo XX, con lo cual la relación entre dirigencia, militancia y gobierno varió, ya que ahora predomina el conflicto por saber si la dirigencia es agente de la militancia o del partido en el gobierno.

Finalmente, en la década de los noventa, a decir de Katz y Mair (2007) se gestó la prevalencia de la cara en el gobierno, de ahí que hablen del partido cartel. Los partidos son concebidos como agentes del Estado y no más como representantes de la sociedad.

Así pues, los partidos cambiaron y se alejaron de lo que mostraron Michels y Duverger, eso fue indudable, aunque como afirman Montero y Gunther (2007), el interés por la vida interna disminuyó, debido a que emergieron nuevos temas de interés, tal como: el comportamiento electoral o el papel de los partidos en el gobierno. Y es que, por un lado, aconteció el cambio de una democracia de masas a una electoral, mientras que, por otro lado, emergieron y tuvieron su auge otras miradas de análisis de los partidos. Aunado a ello, las principales problemáticas con los modelos de partido son su incapacidad, dada su escasez, longevidad y contexto en

\footnotetext{
${ }^{6}$ Para Michels una de las causas que genera la oligarquización es la prevalencia de una militancia desinteresada de la política que idolatra líderes y "está encantada de encontrar personas que se tomen la molestia de atender las cuestiones (internas)" (MICHELS, 1983, p. 97 y 98). A lo anterior, hay que sumar el hecho de que la masa tiene una enorme gratitud "hacia quienes hablan o escriben en su defensa" (MICHELS, 1983, p. 103).
} 
el cual emergieron, para dar cuenta de la variedad de partidos surgidos de la tercera ola democratizadora. Por ende, la sugerencia es retomarlos con suma cautela ${ }^{7}$.

\section{La democracia interna y sus distintas miradas}

El abandono del interés en torno a la concentración de poder fue recuperado gracias al cúmulo de trabajos alrededor del concepto de democracia interna. En general, dicha perspectiva se ha decantado en tres vías que podemos resumir como los estudios interesados en lo que debe, puede y es la organización interna. Pese a ello, el gran ausente en la mayoría de los trabajos es el militante, sea por cuestiones metodológicas (ya que al subir el número de observaciones y/o dimensiones relativas a la democracia interna se reduce la profundidad (ESPEJEL, 2013) o por el lugar en que se observa la democracia interna (ya que los dos primeros tipos de trabajos solo atienden al ámbito estatutario y no la realidad organizativa). En todo caso, vale la pena apreciar los aportes y limitantes de esta forma de abordar la vida intrapartidaria.

El abandono del interés por los partidos en nuestra realidad latinoamericana, se vio reforzado por la tardía entrada a la democracia en la región, vía la tercera ola, lo cual condujo a que el estudio de los partidos se enfocara en aspectos diferentes a la vida interna, centrándose más bien en el ambiente electoral. Ya que las estructuras partidarias enfocaban sus baterías en la obtención del poder, aunado a que en buena parte de la región la existencia de partidos dominantes, la proscripción de otros o la reciente emergencia de la oposición volvía hasta cierto punto irrelevante enfocarse en la vida interna de los partidos con poco tiempo en el espectro político (FREIDENBERG y ALCÁNTARA, 2003).

Por consiguiente, a mediados de la década de 1990 los estudios sobre la vida al interior de los partidos no eran la constante en América Latina, México no era la excepción. Sin embargo, a inicios del siglo XXI emergió un importante esfuerzo de análisis que se enmarcó bajo el concepto de democracia interna. En nuestra región el interés derivó del hecho de que los partidos habían cobrado relevancia, en términos de los resultados y cargos obtenidos, por lo cual desde la academia comenzó interesar el estudio de su vida interna, ya que se trataba de organizaciones clave en el engranaje de las democracias de reciente creación.

\footnotetext{
${ }^{7}$ Por ende, valdría la pena enfatizar que pese a la brillantez de la obra de Michels y Duverger, en parte son deudoras de su tiempo, por consiguiente no debemos perder de vista que la simple puesta en marcha sin su correcta contextualización espacio temporal podría llevar a equívocos virtuosos (en el caso de Duverger y el militante del partido de masas) o defectuosos (en el caso de Michels y el militante que abona a la oligarquía).
} 
En el caso mexicano, emergió un genuino interés académico con la llegada de la alternancia en el año 2000, acompañada del afianzamiento de un sistema de partidos competitivo. En ese tenor de ideas, la literatura relativa a la democracia interna en México puede agruparse bajo dos directrices. En ambos casos, vale la pena mencionarlo, se ha incurrido en el descuido de dar poca evidencia empírica sobre el militante, de "carne y hueso", al concentrar sus baterías en el accionar poco democrático de los dirigentes y/o en la exigencia de mecanismos estatutarios que permitan el empoderamiento de la militancia.

En ese sentido, los trabajos pueden agruparse de acuerdo con el criterio que utilizan para observar la democracia interna. Por ello, existen trabajos jurídicos que intentan dilucidar la democracia interna a través del ámbito estatutario (estudios sobre lo que debe y puede), apelando por su reforma en el caso de partidos que legislen al respecto. Mientras que en segunda instancia existen trabajos politológicos que se centran en la realidad partidaria (estudios sobre lo que es) que retoman cautelosamente las reglas, dirigiendo su mirada a lo que sucede en los procesos de toma de decisiones (ESPEJEL, 2013)

$\mathrm{Al}$ interior de los trabajos jurídicos se encuentran los que enfocados en el debe ser, los cuales defienden la necesidad de contar con la protección de derechos fundamentales de la militancia, su participación en la toma de decisiones y la existencia de mecanismos de control de los dirigentes (NAVARRO, 1999). Este tipo de trabajo es prolijo en plantear un catálogo de derechos y libertades que debe poseer la militancia al interior de los partidos.

Sin duda, este tipo de trabajos poseen valor intrínseco ya que su mirada prescriptiva nos da una guía sobre cómo deberían acontecer los procesos de toma de decisiones. No obstante, su alcance es limitado en la medida que no evalúan la realidad partidaria y, por ende, ofrecen una imagen idílica y homogénea del militante. Junto a este tipo de análisis se encuentran los interesados por evaluar el ámbito estatutario. Se trata de una primera generación de trabajos evaluativos, antes que prescriptivos.

Los trabajos considerados optan por dos caminos. En primera instancia, aumentar los casos con el objetivo de comparar, pero reduciendo los elementos relativos a la democracia interna. Es el caso de Scarrow, Webb y Farrell (2004), enfocados en el caso europeo, que analizan la selección de candidatos y dirigentes, así como la formulación de plataformas en dieciocho democracias, clarificando que los procesos son cada vez más inclusivos, pero no de un modo que restrinja a los líderes 
por completo. Otro ejemplo es el trabajo de Freidenberg (2006), en el cual se muestra que a pesar de la tendencia a la inclusividad en los procesos internos, aún sigue siendo importante el papel de los órganos ejecutivos colegiados en América Latina.

En segundo lugar, otros trabajos prefieren reducir el número de casos, ya se centrándose en un sistema político o en pocos partidos del mismo, para concentrarse en un mayor número de elementos relativos a la democracia interna. Es el caso el trabajo de Calleja (2000) sobre el nivel estatutario del Partido de la Revolución Democrática (PRD), en el que analiza veinticinco variables en el plano estatutario y se determina que tiene "una tendencia casi nula a la oligarquía" (CALLEJA, 2000, p. 93).

Nuevamente la limitante estriba en no considerar que algunas veces lo que dicen los estatutos puede estar muy alejado de la realidad partidaria. De ahí que, sea necesario trascender al análisis sobre lo que es la democracia interna. En ese sentido, el segundo bloque de estudios politológicos tiende a centrarse en el análisis de procesos de toma de decisiones, tal como selección de dirigentes y/o candidatos.

De igual forma, existen dos tipos de caminos. Primero, aquellos que optan por aumentar el número de casos, reduciendo el número de indicadores (trabajando normalmente la selección de candidatos). A nivel internacional, resalta el trabajo de Chambers y Croissant (2008), centrado en el caso asiático, en el cual concluyen que en los tres principales partidos de Tailandia las decisiones siguen siendo tomadas por el líder del partido y/o un grupo cerrado. En América Latina, los trabajos de Aquino (2004) y Picado (2009) dan cuenta, respectivamente de los casos de República Dominicana y Costa Rica. En el primer caso se muestra que los partidos centralizados (Partido de la Liberación Dominicana) fueron más exitosos que los descentralizados (Partido Reformista Social Cristiano y Partido Revolucionario Dominicano) en la puesta en marcha de primarias para candidato presidencial. En el segundo trabajo se analizan los procesos de selección del candidato presidencial y diputados en Costa Rica, así como los factores que incentivaron adoptar mecanismos inclusivos, tal como: reformas electorales, efecto de imitación de otros partidos y presión de organismos internacionales.

Mientras que, en el caso mexicano, resaltan los trabajos que estudian diversos partidos. Tal como el de Reveles (2008), en el que da evidencia sobre la inexistencia de apertura a la militancia en la hechura de las plataformas en el Partido de la Revolución Democrática (PRD) y el Partido Acción Nacional (PAN) y la reforma estatutaria en el Partido Revolucionario Institucional (PRI), en la antesala de la elección presidencial de 2006. De igual forma, el trabajo de Prud'homme (2007) que 
afirma que la génesis partidaria es importante para entender el modelo de partido que se configura en su derrotero histórico con su consecuente presencia/ausencia de democracia interna. Así como el trabajo de Sarabia (2011) sobre los procesos de selección de candidatos a presidencias municipales en Ciudad Juárez, en el que da evidencia sobre que los partidos son organizaciones pragmáticas volcadas a los tiempos electorales, más que la representación de intereses sociales o de sus militancias.

Además, existen trabajos que se enfocan en un caso (partido), aunque centrándose en pocos indicadores (selección de candidato presidencial y/o dirigente nacional), gracias a lo cual logran mayor profundidad mostrando los conflictos, actores y lógica subyacente a dichos procesos.

El libro coordinado por Corona y Casanova (2013), incluye trabajos sobre el PAN (REVELES, 2013), PRD (BOLÍVAR, 2013) y el PRI (ZAMITIZ, 2013), da cuenta de la prevalencia de los dirigentes sobre los militantes en torno a los procesos de selección de candidatos y/o dirigentes. Mientras que la obra coordinada por Corona (2014), contiene artículos sobre el Partido Verde Ecologista de México (PVEM) (ESPEJEL, 2014), el Partido del Trabajo (PT) (DÍAZ, 2014), Movimiento Ciudadano (MC) (BOLÍVAR, 2014), en los que se muestra la configuración de organizaciones cerradas al peso de la militancia en los procesos de toma de decisiones.

Ahora bien, este tipo de estudios centrados en la realidad partidaria permiten, a diferencia de los centrados solamente en el nivel estatutario, apreciar los diversos actores en conflicto, la importancia del origen, el papel de los dirigentes, así como la modificación de las elecciones internas y algunos factores que propician el cambio, tal como los triunfos o las derrotas electorales.

No obstante, en ambos casos, es decir tanto la mirada jurídica como la politológica, al hablar del militante subyace cierta idealización sobre el mismo, además de presuponer su homogeneidad entre distintos partidos, pues se asume que el militante debe contar con mecanismos de participación en la toma de decisiones y control de dirigentes, salvaguardados en los estatutos, para que el partido cuente con procesos democráticos, sin preguntarse si existe una militancia interesada en ello, por ejemplo.

Así pues, en este tipo de abordaje centrado en la democracia interna se ha puesto mucha atención al papel y peso de los dirigentes, dejando de lado la visión del militante, su voz y experiencia en cuanto a su relación con el dirigente. Es decir, se ha estudiado un solo lado de la moneda, asumiendo implícitamente que la militancia ha 
permanecido inalterada a los cambios en la sociedad, la democracia y los partidos. Además, al dejar fuera al militante y mostrar escasa evidencia empírica sobre su accionar y/o sobre su percepción sobre sus funciones al interior de los partidos, ha prevalecido una mirada idílica y alejada de la realidad.

\section{E1 (escaso) estudio de las militancias}

Respecto al estudio de las militancias, se debe indicar que este tipo de investigaciones es variado y reciente, el contexto mayormente estudiado es el europeo, aunque normalmente planteado como un dilema de eficiencia partidista. De cualquier forma se trata de trabajos que dan evidencia empírica del perfil, peso y papel de las militancias en las organizaciones partidistas, con lo cual se logra una visión más compleja de las organizaciones partidistas. Algunos estudios son de largo alcance en cuanto al número de observaciones, en otros se estudian casos en profundidad.

En cuanto al primer bloque, centrado en el caso europeo, existen trabajos que dan cuenta de la incongruencia ideológica de las militancias, lo cual deriva de individuos políticamente más interesados y con capacidad de incidir en las decisiones, los cuales son más propensos a evaluar negativamente a su dirigencia, votar por otro partido o salirse, por ende este fenómeno tiene consecuencias para la competencia electoral (KOLLN y POLK, 2016).

El trabajo de Scarrow y Gezgor (2010) se enfoca en el declive de la militancia partidaria en Europa, pese a la emergencia de mecanismos de participación internos. Por ende, afirman el tránsito del partido de masas de Duverger al "partido del medio", ya que, si bien existe diferencia de edad entre la militancia y la población en general, en dimensiones como el ingreso, la afiliación sindical, la religiosidad y la ideología existen similitudes.

Otros trabajos han revisado la reducción de la militancia partidaria y sus consecuencias para el partido (PONCE y SCARROW, 2014), mostrando que la disminución de la militancia ha afectado la capacidad de los partidos para movilizar en la competencia electoral de 1990 al 2000.

El trabajo de Mair y Van Biezen (2001) se enfoca en el estudio de la reducción de los niveles de participación de las militancias en 20 democracias europeas de 1980 al 2000. Lo cual explican como una manifestación de lo que ocurre con otras formas tradicionales de mediación, tales como iglesias o sindicatos, debido al impacto de la individualización de las preferencias sociales y políticas, así como la 
renuencia a confiar en las estructuras institucionales para representar y articular demandas cada vez más particularizadas.

El trabajo de Hooghe y Kern (2015) se enfoca en el estudio de los partidos en Europa, en dicho contexto no encuentra una relación significativa entre la disminución de la militancia y los niveles de confianza política. Por ende, los partidos siguen funcionando como mecanismos de vinculación, aunque no de corte privilegiado sobre otros, tal como movimientos, organizaciones de la sociedad civil, entre otros.

El trabajo de Wauters (2016) se interesó en explicar por qué los militantes emprenden acciones no institucionalizadas (boicots, peticiones y protestas). Al analizar 22 países europeos encontró que se trata acciones complementarias, en las cuales, los militantes de oposición son más propensos a boicotear productos y firmar peticiones on line (del tipo change.org), mientras que entre más a la izquierda se encuentre el militante, es más propenso a participar en protestas callejeras.

Respecto a los casos en profundidad, existen trabajos que revisan el caso canadiense, mostrando que pocos ciudadanos pertenecen a partidos, debido a la reducción de confianza del ciudadano, y los que pertenecen no son representativos de la población (en edad, género, ocupación e ingreso). Por ello, se trata de organizaciones poco representativas, con pocos miembros y poca vitalidad (CROSS y YOUNG, 2004).

En el caso Irlandés se ha indagado la composición de la militancia en la Familia de los Irlandeses (Fine Gael) (GALLAGHER y MARSH, 2004). Los hallazgos muestran que el militante medio es más viejo, mayormente hombre y en menor medida proveniente de la clase obrera a diferencia del votante medio del partido. Además, casi la mitad de los militantes tienen vínculos con un político individual con el cual se sienten más identificados, antes que al partido.

En el caso de Bélgica (HOOGHE y DASSONNEVILLE, 2012) se muestra que los militantes siguen funcionando como mecanismo de vinculación entre el partido y la sociedad, por ende, la afiliación al partido tiene un fuerte efecto sobre los resultados de las elecciones de 1981 a 2010, aunado a que dicha afiliación se ha mantenido estable.

En torno al mismo contexto, Wauters (2010) estudió la participación de los militantes en las elecciones internas del Partido por la Libertad y el Progreso y el partido Liberales y Demócratas Flamencos. El trabajo pone a prueba diversas teorías, comprobando que las personas participan porque quieren impactar en la política que 
implementará el partido (motivación instrumental). De igual forma muestra que quienes votaron en el pasado son más propensos a hacerlo de nuevo, sobre todo si tuvieron éxito (participación como hábito).

En el caso británico, Fisher, Denver y Hands (2006) analizan el impacto de los resultados electorales sobre la pertenencia y la actividad de la militancia. Mostrando que una espiral de desmovilización puede surgir de la derrota electoral y una espiral de movilización del triunfo, aunque cualquier espiral puede romperse por un resultado electoral dramático con implicaciones organizativas en el corto y largo plazos.

En el mismo contexto, el trabajo de Seyd y Whiteley (2004) se estudió la disminución de la militancia y el activismo en los tres principales partidos de Gran Bretaña. El estudio afirma que la disminución del activismo se debe, del lado de la oferta, al tiempo empleado en el trabajo, en el hogar o el ocio. Mientras que, del lado de la demanda, los partidos tienen menos necesidad de activistas para recaudar fondos y movilizar.

Por otro lado, los trabajos existentes para América Latina pueden dividirse en dos bloques: estudios jurídicos y politológicos.

Respecto el primer bloque, dominan los estudios centrados en un solo caso. Claro ejemplo de ello son los trabajos de Castillo González (2004) y Ramírez Díaz (2014). El primero se centró en el análisis de los litigios presentados por ciudadanos para invocar la protección jurisdiccional electoral frente a actos $\mathrm{u}$ omisiones de sus dirigencias. El trabajo concluye que las tendencias oligarquizadoras conservan fuerte arraigo en México, lo cual se expresa en la incompleta normatividad, la escasa claridad de la regulación interna y el rechazo a la jurisdicción estatal. Mientras el segundo trabajo estudia el nexo entre los derechos humanos y la militancia, a través del análisis de sentencias y resoluciones judiciales; mostrando que el Estado mexicano ha garantizado los derechos de los militantes a través de la vigilancia de los actos partidarios que realiza el Tribunal Electoral del Poder Judicial de la Federación, el cual resuelve normalmente basado en tratados internacionales.

Respecto a los estudios politológicos, existen trabajos de largo alcance, tal como el de Lázaro (2017) que indaga la diversidad de vías de acceso de la militancia en América Latina, la distancia existente entre militantes y dirigentes, el consecuente fin de la militancia y el tránsito a la "independencia política", debido a la crisis de los partidos políticos y el auge de los movimientos independientes. El trabajo de Cozachcow (2018) analiza la importancia de las militancias juveniles en los partidos oficialistas en Argentina y Brasil, así como las consecuencias que conlleva el tránsito a 
la oposición, resaltando la habilitación de procesos de renovación partidaria. La investigación de Piñeiro y Rosenblatt (2017) indaga sobre los tipos de activistas (leales, leales ambiciosos, ambiciosos) en partidos políticos de Chile, Costa Rica y Uruguay, encontrando que es probable que en un momento dado predomine un tipo de activistas y, años más tarde, otro.

Existen otros trabajos centrados en un solo sistema de partidos, tal como el de Jave y Uchuypoma (2016) que estudia la militancia juvenil en el Partido Aprista Peruano y el Partido Popular Cristiano, en ambos casos da cuenta de las expectativas, motivaciones y demandas de los jóvenes, así como los incentivos (ideología, identidad, poder, status, cargos o dinero) que los partidos les ofrecen y los canales formales e informales de participación.

Respecto al caso mexicano, los trabajos interesados en la realidad partidaria son escasos. El estudio de Muñoz, Heras-Gómez y Pulido-Gómez (2013) sobre partidos minoritarios, en el cual se examinan las motivaciones y el perfil de los militantes del Partido Verde Ecologista de México, el Partido del Trabajo, Convergencia y Nueva Alianza a nivel subnacional (Estado de México, Jalisco, Distrito Federal, Veracruz y Michoacán). El principal hallazgo es que la motivación primordial para enrolarse en un partido tiene que ver con la recepción de incentivos materiales.

El trabajo de corte histórico de Martínez Valle (1999) enfocado en indagar la militancia panista de corte católico, así como su influencia en la definición de las metas ideológicas y las estrategias políticas, de 1939 a 1962.

La investigación de Medrano y Muñoz (2013) cuyo objetivo es observar qué tanta incidencia tiene la militancia en la formulación de políticas públicas, una vez que un candidato de su partido asume el gobierno. Al respecto, muestran las percepciones de la militancia prí́sta respecto de las relaciones partido-gobierno en un caso subnacional (Estado de México). El principal hallazgo es que la militancia solicita mayor partidismo en las decisiones políticas que en la configuración del gabinete.

Finalmente, el trabajo de Díaz y Espejel (2018) da cuenta de la opinión de los militantes sobre la vida interna en el partido Movimiento Regeneración Nacional. La investigación muestra que el militante percibe que no se le toma en cuenta en las principales decisiones del partido, tal como seleccionar dirigentes nacionales o candidatos presidenciales. 
Como es evidente, el estudio de las militancias muestra grandes avances en democracias consolidadas, no así en el contexto latinoamericano, mucho menos en México. Por consiguiente, es pertinente alentar el estudio de las militancias en contextos cercanos al nuestro, para no caer en idealizaciones como sucede con la mayoría de los trabajos referidos a la democracia interna y, con ello, atender el importante esfuerzo analítico de comprender al partido por dentro que inauguraron los estudios organizacionales.

\section{A manera de conclusión}

El estudio de las militancias fue, hasta cierto punto, desatendido en la medida de que el foco de atención se centró en el centro de poder que subyacía a cualquier organización, lo cual cobró sentido a través de diversos modelos de partido en el siglo XX. No obstante, el interés que emergió bajo el concepto democracia interna y que derivó en una serie de trabajos de corte jurídico y politológico tuvo a bien poner nuevamente la atención en la vida al interior de los partidos. Empero, el descuido fue concentrarse en los dirigentes, dejando de lado a los militantes, presuponiendo con ello su homogeneidad entre partidos.

A la par de la consolidación de los trabajos sobre democracia interna, emergió un esfuerzo por estudiar a las militancias en democracias consolidadas, dicho esfuerzo comienza a tener frutos en la región latinoamericana. Pese a ello, sigue siendo reducido el interés por el estudio de la militancia, con lo cual se está dejando de lado a uno de los actores primordiales en las organizaciones partidistas. Con lo anterior, parece que la idea que subyace en las investigaciones sobre partidos es la siguiente: para entender cómo funcionan los partidos hay que describir y/o explicar cómo actúan o se agrupan los dirigentes. Por ende, es pertinente revitalizar el interés por la militancia, tal fue la intención del presente trabajo, ya que en el contexto latinoamericano se ha explorado poco el perfil, accionar y percepción del militante al interior de los partidos (un actor poco valorado en la literatura general de los partidos, así como idealizado por la literatura particular de la democracia interna).

En ese sentido, vendría bien relacionar el interés de los estudios sobre democracia interna con los trabajos centrados en la militancia, ya que ello permitirá analizar el papel de la militancias sobre la toma de decisiones al interior del partido, ofreciendo evidencia empírica que dé cuenta de la militancia realmente existente. Y es que, al obviar o idealizar al militante y, por ende, pasar por alto las percepciones y acciones del mismo, se ha producido una visión parcial del funcionamiento de los partidos. 
Tomando en cuenta lo anterior, a continuación se plantean algunas ideas/hipótesis que podrían explorarse en el caso mexicano, tomando en cuenta el interés de explorar al militante y su relación con la democracia interna, así como la historia y el contexto partidista:

- $\quad$ Si, derivado de la génesis partidaria, en partidos con ideologías conservadoras, la militancia tiende a legitimar decisiones cupulares ${ }^{8}$.

- $\quad \mathrm{Si}$, en virtud del origen partidario, en partidos del espectro político de la izquierda, la militancia tiende a ser más proclive al debate y conflictos internos?.

- $\quad$ Si las reglas del juego interpartidarias importan $y$, por consiguiente, los partidos minoritarios y/o emergentes tienen menores alicientes para permitir una mayor participación de los militantes en la toma de decisiones internas, debido a que su principal preocupación es mantener el registro oficial ${ }^{10}$.

Si las reglas del juego intrapartidarias importan y, por consiguiente, la escasa participación del militante en la toma de decisiones deriva de los requisitos de entrada, permanencia, así como derechos y obligaciones estipulados en los estatutos partidarios ${ }^{11}$.

\footnotetext{
${ }^{8}$ Vale la pena recordar el énfasis en las jerarquías partidarias que tuvo en su génesis el PAN (1939), por lo cual tuvieron que pasar sesenta ańos para que la militancia pudiera seleccionar a su candidato presidencial, pues previamente lo hacía el Consejo Nacional (integrado por no más de 350 personas).

9 Por ejemplo, el PRD, de centro izquierda, se ha caracterizado por vivir procesos de selección de dirigentes muy complicados, de tal suerte que los medios de comunicación han caracterizado a los grupos internos como tribus.

${ }^{10}$ Dependiendo del sistema electoral, el umbral para mantener el registro varía. En México, a partir de 2014 se acrecentó a 3\%, lo cual derivó en la desaparición de dos partidos minoritarios (Partido Encuentro Social y Partido Nueva Alianza), mismos que se caracterizaron por no consultar a la militancia la selección de candidatos en 2018.

11 Por ejemplo, el PAN mantuvo una restricción importante hasta 2015, ya que exigía que todo militante fuera previamente, al menos por 18 meses, adherente. La diferencia era que solo el militante activo podía incidir en las decisiones partidistas. En cambio, el partido Movimiento Regeneración Nacional solo considera la figura de afiliado, el cual puede incidir en las decisiones internas sin pasar por algún filtro.
} 
- $\quad$ Si existe una militancia autointeresada, para la cual la toma de decisiones internas resultan poco importantes, salvo que ello suponga obtener mayores incentivos selectivos (puestos, cargos, dinero etc.) ${ }^{12}$.

- $\quad$ Si al interior de un partido perviven distintas subculturas que generan mayor conflicto, por ejemplo, entre posiciones radicales y moderadas en el seno de la izquierda política ${ }^{13}$.

Es evidente que cada uno de estos puntos puede complejizarse. No obstante, la idea es sembrar una semilla que aliente estudios empíricos donde el interés se centre en el militante. Por lo cual, la intención de dichos trabajos tendría que ser proporcionar evidencia empírica sobre el perfil, el papel y el peso de la militancia el interior de los partidos.

- Alberto Espejel Espinoza é Doutor em Ciência Política pela Universidade Nacional Autônoma do México (UNAM). Professor investigador da Faculdade de Estudos Superiores Acatlán. Integrante do Comitê Diretivo e Membro fundador da Revista Electrónica Posibilidad Política. E-mail: alberto.espejel.espinoza@ gmail.com.

\section{Referências}

AQUINO, José. Partidos políticos y elecciones primarias: construyendo la democracia interna. Ciencia y Sociedad, v. XXIX, n. 3, p. 405-425, 2004.

\footnotetext{
${ }^{12}$ La investigación de Muńoz, Heras-Gómez y Pulido-Gómez (2013), previamente citada, da cuenta de militantes autointeresados que se activan fundamentalmente cuando se les ofrecen incentivos materiales.

${ }^{13}$ Una de las pugnas programáticas añejas del PRD fue la suscitada entre quienes estaban abiertos al diálogo con otros partidos en el legislativo o con el gobierno en turno (moderados) frente a quienes planteaban que las causas del partido se conseguirán con apoyo de organizaciones sociales y en las calles. Dicha pugna se ha trasladado a procesos de selección de dirigentes derivando en elecciones ríspidas, tal como en 2008 en que Alejandro Encinas representaba el ala radical y Jesús Ortega la moderada.
} 
BOLÍVAR, Rosendo. La oligarquización del partido de la revolución democrática bajo la dirigencia de Nueva Izquierda. In: CORONA, Gabriel; CASANOVA, Francisco (Orgs.). Democracia interna y tendencias oligárquicas de los partidos políticos en México: PAN, PRI y PRD, México: Gernika, 2013, p. 229-274.

BOLÍVAR, Rosendo. Movimiento Ciudadano: democracia interna y tendencias oligárquicas. In: CORONA, Gabriel (Org.). Democracia interna y tendencias oligárquicas de los partidos políticos en México: Partido Verde Ecologista, Partido del Trabajo, Movimiento Ciudadano y Nueva Alianza, México: Gernika, 2014, p. 277-340.

CALLEJA, Jennifer. Democracia interna y externa: el caso del PRD. Espiral, v. VII, n. 19, p. 71-93, 2000.

CASTILLO, Leonel, Los derechos de la militancia partidista de la jurisdicción. México: Tribunal Electoral del Poder Judicial de la Federación, 2004.

CHAMBERS, Paul; CROISSANT, Aurel. Intra-party democracy in Thailand. Unpublished manuscript, $2008 \quad$ Disponível em: http://www.uniheidelberg.de/imperia/md/content/fakultaeten/wiso/ipw/croissant/publications/chambers_croissant_ 2008_intraparty_democracy.pdf>. Acesso em: 21 nov. 2017.

CORONA, Gabriel (Org.). Democracia interna y tendencias oligárquicas de los partidos políticos en México: Partido Verde Ecologista, Partido del Trabajo, Movimiento Ciudadano y Nueva Alianza, México: Gernika, 2014, p. 277-340.

CORONA, Gabriel; CASANOVA, Francisco (Orgs.). Democracia interna y tendencias oligárquicas de los partidos políticos en México: PAN, PRI y PRD, México: Gernika, 2013, p. 311-438.

COZACHCOW, Alejandro. Militancias juveniles y oficialismo son América Latina. Algunas reflexiones sobre los vínculos entre juventudes y partidos políticos en el gobierno en Argentina y Brasil entre 2001 y 2016. Encrucijadas. Revista Crítica de Ciencias Sociales, v. 15, p. 1-21, 2018.

CROSS, William; YOUNG, Lisa. The Contours of Political Party Membership in Canada. Party Politics, v. 10, n. 4, p. 427-444, 2004.

DÍAZ, Mariela. Proceso de selección de candidatos en el Partido del Trabajo: un análisis de sus estatutos y de las elecciones de 2006 y 2012. In: CORONA, Gabriel (Org.). Democracia interna y tendencias oligárquicas de los partidos políticos en México: Partido Verde Ecologista, Partido del Trabajo, Movimiento Ciudadano y Nueva Alianza, México: Gernika, 2014, p. 205-240.

DÍAZ, Mariela; ESPEJEL, Alberto. Militancia partidaria y toma de decisiones en el Movimiento Regeneración Nacional. Revista Mexicana de Estudios Electorales, v. 2, n. 20, p. 159-193, 2018.

DOWNS, Anthony. Teoría económica de la democracia. Madrid: Aguilar, 1973.

DUVERGER, Maurice. Los partidos políticos. México: Fondo de Cultura Económica, 1984.

ESPEJEL, Alberto. El estado de la cuestión de la democracia interna. In: CORONA, Gabriel; CASANOVA, Francisco (Orgs.). Democracia interna y tendencias oligárquicas de los partidos políticos en México: PAN, PRI y PRD, México: Gernika, 2013. p. 31-68.

ESPEJEL, Alberto. La concentración de poder en el liderazgo dominante. La (ausencia) de democracia interna del Partido Verde Ecologista de México (1993-2012). In: CORONA, Gabriel (Org.). Democracia interna y tendencias oligárquicas de los partidos políticos en México: Partido Verde Ecologista, Partido del Trabajo, Movimiento Ciudadano y Nueva Alianza, México: Gernika, 2014, p. $87-130$. 
. La democracia interna de los principales partidos en México. 338f. Un acercamiento al origen y derroteros del PRI, el PAN y el PRD. México: UNAM (tesis de doctorado), 2015.

FISHER, Justin; DENVER, David; HANDS, Gordon. Party Membership and Campaign Activity in Britain. Party Politics, v. 12, n. 4, p. 505-519, 2006.

FREIDENBERG, Flavia. La democratización de los partidos políticos: entre la ilusión y el desencanto. In: THOMPSON, John; SÁNCHEZ, Francisco (Orgs.). Fortalecimiento de los partidos políticos en América Latina: institucionalización, democratización y transparencia. San José de Costa Rica: Instituto Interamericano de Derechos Humanos, 2006, p. 91-141.

FREIDENBERG, Flavia; ALCÁNTARA, Manuel. Organización y funcionamiento interno de los partidos políticos en América Latina. In: FREIDENBERG, Flavia; ALCÁNTARA, Manuel (Orgs.). Partidos Políticos de América Latina. México: Fondo de Cultura Económica, 2003, p. 1-17.

GALLAGUER, Michael; MARSH, Michael. Party Membership in Ireland. Party Politics, v. 10, n. 4, p. 407-425, 2004.

HOOGHE, Marc; KERN, Anna. Party membership and closeness and the development of trust in political institutions: An analysis of the European Social Survey, 2002-2010. Party Politics, v. 21, n. 6, p. 944-956, 2015.

HOOGHE, Marc; DASSONNEVILLE, Ruth. Party members as an electoral linking mechanism. Party Politics, v. 20, n. 3, p. 368-380, 2012.

JAVE, Iris; UCHUYPOMA, Diego. Jóvenes y partidos políticos. Dinámicas de la militancia en el APRA y el PPC. Perú: Konrad Adenauer Stiftung, 2016.

KATZ, Richard; MAIR, Peter. La supremacía del partido en las instituciones públicas: el cambio organizativo de los partidos en las democracias contemporáneas. In: MONTERO, Ramón; GUNTHER, Richard; LINZ, Juan (Orgs.). Partidos políticos. Viejos conceptos, nuevos retos. Madrid: Trotta, 2007, p. 101-125.

KIRCHHEIMER, Otto. El camino hacia el partido de todo el mundo. In: LENK, Kurt; NEUMANN, Franz (Orgs.), Teoría y sociología críticas de los partidos políticos. Barcelona: Anagrama, 1980, p. 328-347.

KÖLLN, Ann-Kristin; POLK Jonathan. Emancipated party members. Party Politics, v. 23, n. 1, p. 18-29, 2016.

LÁZARO, Gerardo. La informalización de la política: de la militancia política la independencia política. Acta Científica, s/n. 2017. Disponível em: <http://actacientifica.servicioit.cl/biblioteca/ gt/GT10/GT10_LazaroG.pdf>. Acesso em: 17 septiembre 2018.

MAIR, Peter; VAN BIEZEN, Ingrid. Party membership in twenty european democracies, 1980-2000. Party Politics, v. 7, n. 1, p. 5-21, 2001.

MARTÍNEZ, Adolfo. Los militantes católicos y el PAN: una historia política, 1939-1962. Este País, n. 102, p. 1-22, 1999.

MARTÍNEZ, Víctor. Partido cartel Una revisión crítica del concepto. Foro Internacional, v. LVI, n. 4, p. 1053-1087, 2016.

MEDRANO, Ramiro y Aldo MUÑOZ. Las percepciones de la militancia respecto de las relaciones partido-gobierno en el Estado de México. México: Instituto Electoral del Estado de México, 2013.

MICHELS, Robert. Los partidos políticos: Un estudio sociológico de las tendencias oligárquicas de la democracia interna. Tomo I y II. Buenos Aires: Amorrortu, 1983. 
MONTERO, Ramón; GUNTHER, Richard. Introducción: Los estudios sobre los partidos políticos. In. MONTERO, Ramón; GUNTHER, Richard; LINZ, Juan (Orgs.). Partidos políticos. Viejos conceptos y nuevos retos, Madrid: Trotta, 2007, p. 15-48.

MUÑOZ, Aldo, HERAS-GÓMEZ, Leticia y Amalia PULIDO-GÓMEZ. Una aproximación a la militancia partidista en México: el caso de los partidos emergentes. Convergencia, n. 63, p.177-205, 2013.

NAVARRO, José. Partidos políticos y "democracia interna". Madrid: Centro de Estudios Políticos y Constitucionales, 1999.

OSTROGORSKI, Moisei. Democracy and the organization of political parties. Volume I: England. London: Transaction Books, 1982.

PANEBIANCO, Angelo. Modelos de partido. Madrid: Alianza Universitaria, 1990.

PICADO, Hugo. Cuando las primarias son secundarias. Causas y efectos de los modos de selección de candidatos en el PLN y el PUSC (1990-2006). In: FREIDENBERG, Flavia; ALCÁNTARA, Manuel (Orgs.). Selección de candidatos, política partidista y rendimiento democrático. México: TEDF, 2009, p. 209-240.

PIÑEIRO, Rafael; ROSENBLATT, Fernando. Tipos de activistas sonorenses partidarias. Política $y$ Gobierno, v. XXIV, n. 2, p. 275-300, 2017.

PONCE, Aldo; SCARROW, Susan. Which members? Using cross-national surveys to study party membership. Party Politics, v. 22, n. 6, p. 679-690, 2014.

PRUD'HOMME, Jean-Francoise. La vida interna de los partidos mexicanos y la democracia (20002003). In. CASTAÑOS, Fernando; LABASTIDA, Julio; LÓPEZ-LEYVA, Miguel (Orgs.). El estado actual de la democracia en México: retos, avances y retrocesos, México: UNAM, 2007, p. 245-280.

RAMÍREZ, Erwin. Derechos humanos y militancia partidista. México: Tribunal Electoral de Poder Judicial de la Federación, 2014.

REVELES, Francisco. La democracia en los partidos políticos: Premisas, contenidos y posibilidades. México: Instituto Electoral del Estado de México, 2008.

- La coalición dominante del Partido Acción Nacional como partido gobernante. In. CORONA, Gabriel; CASANOVA, Francisco (Orgs.). Democracia interna y tendencias oligárquicas de los partidos políticos en México: PAN, PRI y PRD, México: Gernika, 2013, p. 123-150.

SARABIA, Cecilia. La selección de candidatos a la presidencia municipal de los partidos políticos en Ciudad Juárez como práctica y reforzamiento democracia interna. Estudios Fronterizos, v. 12, n. 24, p. 159-183, 2011.

SARTORI, Giovanni. Partidos y sistemas de partidos. Madrid: Alianza, 1980.

SCARROW, Susan; GEZGOR, Burcu. Declining memberships, changing members? European political party members in a new era. Party Politics, v. 16, n. 6, p. 823-843, 2010.

SCARROW, Susan; WEBB, Paul; FARRELL, David. De la integración social a la competición electoral. La nueva distribución del poder en los partidos políticos. Zona Abierta, n. 108-109, p. 111153, 2004.

SEYD, Patrick; WHITELEY, Paul. British party members. An overview. Party Politics, v. 10, n. 4, p. 355-366, 2004.

VON BEYME, Klaus. Los partidos políticos en las democracias occidentales. Madrid: Centro de Investigaciones Sociológicas, 1986. 
142 | Alberto Espejel Espinoza

WAUTERS, Bram. Explaining participation in intra-party elections. evidence from belgian political parties. Party Politics, v. 16, n. 2, p. 237-259, 2010.

Which party members participate in direct political action? A cross-national analysis. International Political Science Review, n. 1, p. 1-17, 2016.

ZAMITIZ, Héctor. Una interpretación sobre las tendencias recientes de dirección y control de la oligarquía en el Partido Revolucionario Institucional. In: CORONA, Gabriel; CASANOVA, Francisco (Orgs.). Democracia interna y tendencias oligárquicas de los partidos políticos en México: PAN, PRI y PRD, México: Gernika, 2013, p. 153-194.

Texto recebido em $1^{\circ}$ de maio de 2018 . Aprovado em 29 de outubro de 2018. 